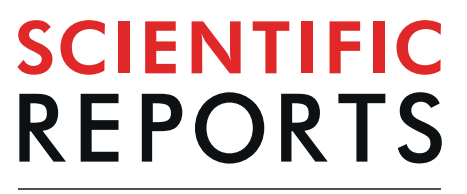

natureresearch

\title{
OPEN The Novel Notch-induced Long Noncoding RNA LUNAR1 Determines the Proliferation and Prognosis of Colorectal Cancer
}

\author{
Zixi Zhang ${ }^{1,2}$, Gai Li ${ }^{1}$, He Oiu ${ }^{1}$, Jingyi Yang ${ }^{3}$, Xin Bu ${ }^{4}$, Shaojun Zhu ${ }^{5}$, Jianyong Zheng ${ }^{6}$, \\ Chengxue Dang ${ }^{7}$, Weizhong Wang ${ }^{6} \&$ Dake Chu $^{1 *}$
}

In contrast to what is known about the complicated roles of Notch signalling in human malignancies, the direct target genes of Notch signalling are still unclear. Recently, long noncoding RNAs (IncRNAs) have been found to play various roles in the post-transcriptional regulation of gene expression. In the present study, we investigated the potential role of the Notch-induced IncRNA LUNAR1 in colorectal cancer (CRC). We recruited 196 cases of clinical CRC specimens and investigated LUNAR1 levels in these specimens. The associations of LUNAR1 with tumour aggressiveness and clinical outcomes were evaluated. Moreover, the impact of LUNAR1 on the malignant behaviour of tumour cells was tested in cell lines. Significantly increased expression of LUNAR1 in clinical CRC specimens was detected compared with that in matching normal tissues. LUNAR1 expression in CRC was found to be associated with the tumour aggressiveness, disease-free survival and overall survival of patients. The downregulation of LUNAR1 in SW620 cells inhibited cell proliferation, migration, invasion and tumour growth while inducing apoptosis. Moreover, the inhibition of LUNAR1 can significantly suppress IGF1 signalling in CRC. These results indicated that LUNAR1 was increased in CRC and might promote tumour progression. Thus, LUNAR1 may constitute a promising prognostic marker for the clinical management of CRC.

The Notch signalling pathway is an evolutionarily conserved pathway that plays an essential role in the regulation of cell fate in most cells types ${ }^{1}$. To date, four receptors of Notch signalling have been discovered in mammals, including Notch 1 , Notch 2, Notch 3, and Notch $4^{2}$. Notch signalling can either negatively or positively affect cellular processes, including proliferation, differentiation and apoptosis, depending on the cell type ${ }^{3}$. Previous investigations also revealed an interesting role of Notch signalling in human malignant tumours. Notch signalling acts as a tumour suppressor in some malignancies while acting as an oncogene in other neoplasms ${ }^{4,5}$. In the gastrointestinal tract, Notch signalling has been proven to be essential in cell proliferation and carcinogenesis ${ }^{6,7}$. In addition, our previous studies demonstrated that Notch 1 could act as an oncogene in colorectal cancer (CRC) ${ }^{8}$. The expression of the Notch1 intracellular domain in CRC was significantly associated with the overall survival of patients, in which patients with increased levels of Notch1 were more likely to have an unfavourable overall survival ${ }^{9}$. Conversely, Notch2 was identified to play a tumour suppressor role in $\mathrm{CRC}^{10,11}$. In contrast to what is known about the complex roles of Notch signalling, only HES and HEY have been confirmed to be direct target genes of Notch signalling. Therefore, the non-canonical pathway might play critical roles in Notch signalling.

Recently, long noncoding RNAs (lncRNAs) have been found to play an essential role in various molecular pathways, including Notch signalling. Specifically, a Notch-regulated lncRNA, LUNAR1, was demonstrated to be

\footnotetext{
${ }^{1}$ Department of Gastroenterology, the First Affiliated Hospital of Xi'an Jiaotong University, Xi'an, China. ${ }^{2}$ Department of Dermatology, the First Affiliated Hospital of Xi'an Jiaotong University, Xi'an, China. ${ }^{3}$ Information Center, the First Affiliated Hospital of Xi'an Jiaotong University, Xi'an, China. ${ }^{4}$ State Key Laboratory of Cancer Biology and Department of Biochemistry and Molecular Biology, Fourth Military Medical University, Xi'an, Shaanxi, China. ${ }^{5}$ Department of Pathology, Tangdu Hospital, the Fourth Military Medical University, Xi'an, Shaanxi, China. ${ }^{6}$ Department of Gastrointestinal Surgery, Xijing Hospital of Digestive Diseases, the Fourth Military Medical University, Xi'an, China. ${ }^{7}$ Department of Surgical Oncology, the First Affiliated Hospital of Xi'an Jiaotong University, Xi'an, China. *email: chudake@xjtu.edu.cn
} 
a key downstream target gene in human T cell acute lymphoblastic leukaemia (T-ALL). LUNAR1 was found to be a 491-nucleotide transcript located at 15q26.3, with 4 exons and a poly (A) tail. This lncRNA was suggested to be required for efficient T-ALL growth in vitro and in vivo ${ }^{12}$. However, the molecular regulation in solid tumours is quite different from that in leukaemia. Therefore, the role of the lncRNA LUNAR1 in solid tumours remains to be determined to clarify the contradiction between the complex roles and limited target genes of Notch signalling.

In the present study, we investigated the potential function and subsequent mechanism of the Notch-regulated lncRNA LUNAR1 in human CRC to gain an understanding of lncRNA-mediated non-canonical Notch signalling in human malignancy.

\section{Results}

Detection of LUNAR1 expression in the study cohort. The characteristics of the 196 patients involved in the study are shown in Table 1. After normalization to the expression of $18 \mathrm{~S}$ rRNA, LUNAR1 expression in CRC was significantly increased in $86.7 \%(170 / 196)$ of clinical CRC specimens compared with that in adjacent normal tissues (Fig. 1A). This increased expression pattern indicated that aberrant LUNAR1 levels might be associated with CRC carcinogenesis. To facilitate further statistical analysis, we manually assigned patients into two groups: patients with increased LUNAR1 levels $(n=145$, fold change $\geq 2)$ and preserved LUNAR1 levels $(n=51$, fold change $<2$ ) (Fig. 1B).

LUNAR1 expression in CRC was correlated with tumour aggressiveness. According to our classification of LUNAR1 expression, we analysed the association of LUNAR1 expression in CRC with clinicopathological factors. Statistical analysis revealed that the difference in LUNAR1 levels among the normal weight, overweight and obesity groups was significant $(P=0.030)$. Subgroup analysis showed that increased LUNAR1 expression was more likely to be detected in obese patients compared with normal weight patients $(P=0.020)$. However, this difference did not reach a statistically significant level between overweight and normal weight patients $(P=0.120)$ or between obese and overweight patients $(P=0.246)$. This association suggested that LUNAR1 might be involved in obesity-related tumourigenesis and progression in CRC. In addition, the expression of LUNAR1 in CRC was also showed to be associated with the tumour differentiation status, invasion depth, node metastasis status and TNM stage, indicating a significant role of LUNAR1 in CRC aggressiveness. No statistically significant association of LUNAR1 with tumour size $(P=0.054)$ or distant metastases $(P=0.161)$ was found. In addition, the associations of LUNAR1 with sex, age, tumour location, MSI, KRAS mutation, BRAF mutation, and PIK3CA mutation were not statistically significant.

LUNAR1 expression in CRC was correlated with the disease-free survival of patients. In the present study cohort, the postoperative median follow-up time of all recruited patients with CRC was 40 months. The association of the LUNAR1 expression level with disease-free survival (DFS) was evaluated by Kaplan-Meier analysis. The univariate analysis results indicated that preserved LUNAR1 expression in CRC was significantly associated with a favourable DFS in patients (Fig. 2, log-rank test: $P<0.001$ ). The median DFS of all the recruited patients was 36.4 months (95\% CI: 29.6-43.2). According to the subgroup classification by LUNAR1 expression level, the DFS of patients with increased LUNAR1 levels was 26.6 months (95\% CI: 20.3.3-32.9); however, DFS could not be estimated in the preserved LUNAR1 expression group due to favourable outcomes. These results indicated that CRC patients with increased LUNAR1 levels were more likely to have tumour recurrence and metastasis. In the Kaplan-Meier analysis, unfavourable DFS was also found to be associated with poor differentiation status (log-rank test: $P<0.001$ ), obesity (log-rank test: $P=0.005)$ and TNM stage (log-rank test: $P<0.001)$. The unadjusted HRs of the clinicopathological factors for DFS are shown in Table 2.

Next, multivariate Cox proportional hazards model analysis adjusted for confounding factors, including sex, age, differentiation status, node status, and TNM stage, was utilized to verify the independent prognostic value of LUNAR1 in patients with CRC. The results of multivariate analysis revealed that preserved LUNAR1 expression in CRC was independently associated with favourable DFS in patients after controlling for known prognostic factors. The adjusted HR for patients with tumours of increased LUNAR1 expression was 2.81 (95\% CI: 1.69-4.67, $P<0.001)$ compared with that of the LUNAR1 preserved group as a reference. The adjusted HRs of the clinicopathological factors for DFS are shown in Table 2.

LUNAR1 expression in CRC was correlated with the overall survival of patients. Considering the association of LUNAR1 with DFS in CRC patients, we next evaluated its correlation with overall survival (OS). As a result, preserved LUNAR1 levels in CRC were significantly associated with favourable OS, similar to its association with DFS (Fig. 3, log-rank test: $P<0.001$ ). The OS time of patients with increased LUNAR1 levels was 33.0 months (95\% CI: 23.3-42.7), but it could not be estimated in patients with preserved LUNAR1 expression because over half of the patients survived 5 years. In the univariate analysis of clinicopathological factors, unfavourable OS was found to be associated with poor differentiation status (log-rank test: $P<0.001$ ), obesity (log-rank test: $P=0.005$ ) and TNM stage (log-rank test: $P<0.001$ ). The unadjusted HRs of the clinicopathological factors for OS are shown in Table 3.

In multivariate analysis, increased LUNAR1 levels were characterized as an independent unfavourable prognostic factor in patients with CRC after adjusting for known prognostic factors. In CRC patients with increased LUNAR1 expression, the adjusted HR of unfavourable prognosis was 2.79 (95\% CI: $1.56-4.99, P<0.001)$ compared with that of patients with tumours of preserved LUNAR1 expression. The adjusted HRs of the clinicopathological factors for OS are shown in Table 3.

Inhibition of LUNAR1 suppresses tumour cell proliferation and invasion. After transfection of LUNAR1 siRNA, real time PCR confirmed that LUNAR1 expression was the highest in SW620 cells, and significantly decreased in the stably transfected cells compared with control cells (Fig. 4A). Therefore, we selected SW620 


\begin{tabular}{|c|c|c|c|c|}
\hline \multirow[b]{2}{*}{ Variable } & \multirow[b]{2}{*}{$\mathbf{n}$} & \multicolumn{2}{|c|}{ LUNAR1 expression } & \multirow[b]{2}{*}{$P$} \\
\hline & & Increased & Preserved & \\
\hline Total & 196 & 145 & 51 & \\
\hline Sex & & & & $0.151^{*}$ \\
\hline Male & 124 & 96 & 28 & \\
\hline Female & 72 & 49 & 23 & \\
\hline Age at diagnosis & & & & $0.312^{*}$ \\
\hline$\leq 60$ & 145 & 110 & 35 & \\
\hline$>60$ & 51 & 35 & 16 & \\
\hline \multicolumn{5}{|l|}{ BMI } \\
\hline Normal weight & 130 & 89 & 41 & $0.030 *$ \\
\hline Overweight & 42 & 34 & 8 & \\
\hline Obese & 24 & 22 & 2 & \\
\hline Tumour location & & & & $0.090^{*}$ \\
\hline Right & 66 & 45 & 21 & \\
\hline Left & 71 & 59 & 12 & \\
\hline Rectum & 59 & 41 & 18 & \\
\hline Tumour size & & & & $0.054^{*}$ \\
\hline$\leq 3.0 \mathrm{~cm}$ & 46 & 29 & 17 & \\
\hline$>3.0 \mathrm{~cm}$ & 150 & 116 & 34 & \\
\hline Differentiation status & & & & 0.038* \\
\hline Well & 42 & 25 & 17 & \\
\hline Moderate & 89 & 67 & 22 & \\
\hline Poor & 65 & 53 & 12 & \\
\hline Depth of invasion & & & & $0.013 *$ \\
\hline $\mathrm{T}_{1}+\mathrm{T}_{2}$ & 68 & 43 & 25 & \\
\hline $\mathrm{T}_{3}+\mathrm{T}_{4}$ & 128 & 102 & 26 & \\
\hline $\begin{array}{l}\text { Lymph node } \\
\text { metastasis }\end{array}$ & & & & $0.024^{*}$ \\
\hline Absent (N0) & 85 & 56 & 29 & \\
\hline Present (N1-3) & 111 & 89 & 22 & \\
\hline Distant metastasis & & & & $0.161^{\dagger}$ \\
\hline Absent (M0) & 174 & 126 & 48 & \\
\hline Present (M1) & 22 & 19 & 3 & \\
\hline TNM stage & & & & $0.033^{\dagger}$ \\
\hline $\mathrm{I}$ & 31 & 17 & 14 & \\
\hline II & 54 & 39 & 15 & \\
\hline III & 89 & 70 & 19 & \\
\hline IV & 22 & 19 & 3 & \\
\hline MSI & & & & $0.426^{*}$ \\
\hline MSS & 168 & 126 & 42 & \\
\hline MSI-H & 28 & 19 & 9 & \\
\hline KRAS mutation & & & & $0.315^{*}$ \\
\hline$(-)$ & 131 & 94 & 37 & \\
\hline$(+)$ & 65 & 51 & 14 & \\
\hline BRAF mutation & & & & 0192* \\
\hline$(-)$ & 165 & 125 & 40 & \\
\hline$(+)$ & 31 & 20 & 11 & \\
\hline PIK3CA mutation & & & & $0.264^{*}$ \\
\hline$(-)$ & 160 & 129 & 31 & \\
\hline$(+)$ & 36 & 26 & 10 & \\
\hline
\end{tabular}

Table 1. Statistical results of LUNAR1 expression. ${ }^{*} P$ value when expression levels were compared using Pearson $\chi^{2}$ test. ${ }^{\dagger} P$ value when expression levels were compared using Fisher’s exact test.

cells for further investigation. The proliferation of SW620 cells was significantly suppressed by the downregulation of LUNAR1, as measured by the CCK-8 reagent responses in the cell viability assay (Fig. 4B). To further investigate the impact of LUNAR1 on proliferation, colony formation and flow cytometry assays were utilized on LUNAR1 siRNA-transfected cells and control cells. The results showed that the number of cancer cell colonies was decreased 

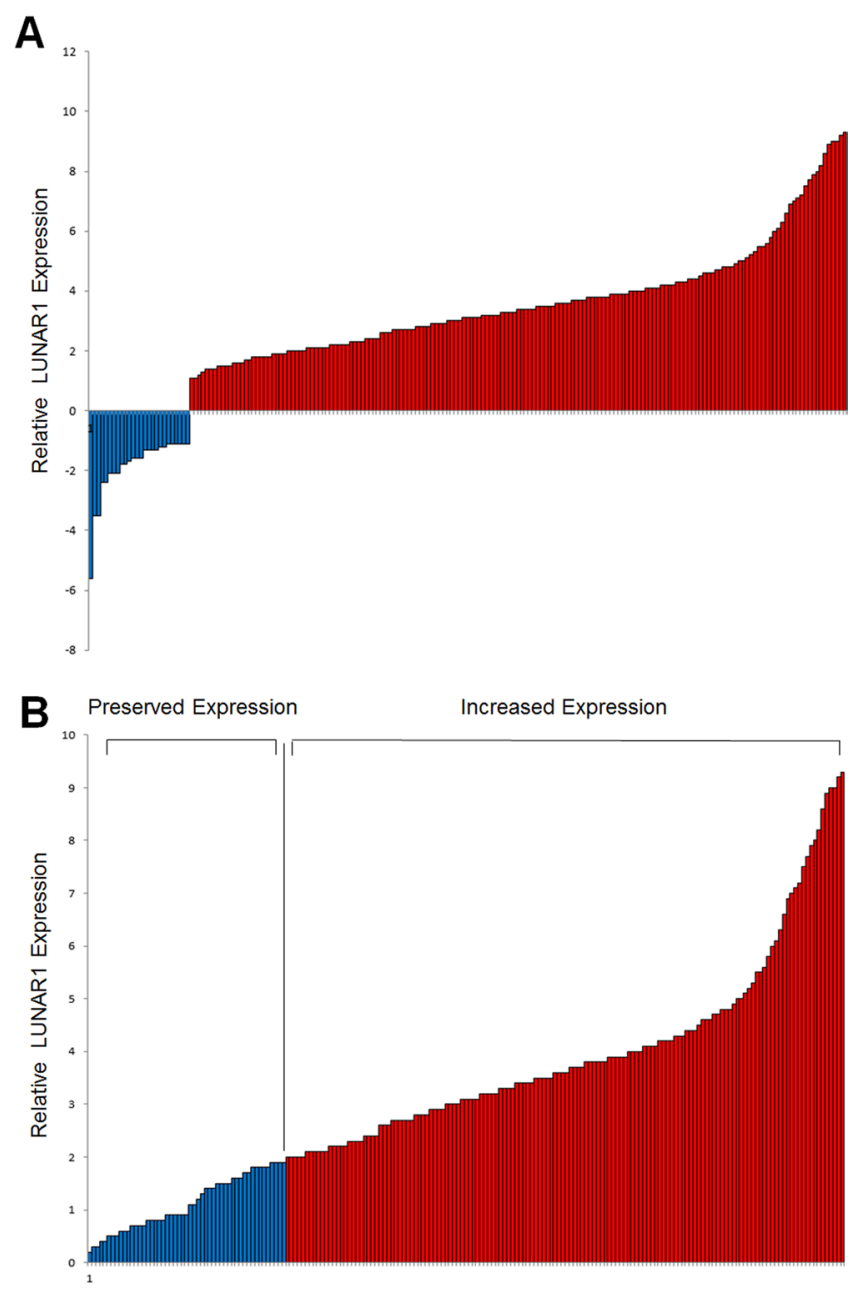

Figure 1. Detection of LUNAR1 expression in clinical CRC specimens. (A) Increased LUNAR1 expression was detected in $170 \mathrm{CRC}$ specimens compared with adjacent normal tissues; (B) Patients were assigned into two groups according to LUNAR1 level: patients with increased LUNAR1 level (fold change $\geq 2$ ) and preserved LUNAR1 level (fold change $<2$ ).

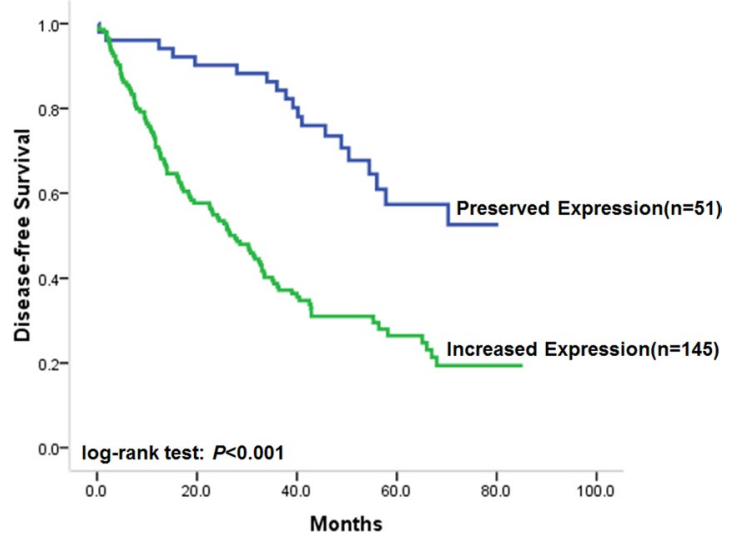

Figure 2. Correlation of LUNAR1 expression with disease-free survival. Kaplan-Meier univariate survival analysis showed that preserved LUNAR1 expression in CRC was significantly associated with favorable DFS of patients.

in LUNAR1-downregulated cells, and LUNAR1 siRNA transfection caused a higher percentage of apoptotic cells (Fig. 4C,D). These results indicated that LUNAR1 could promote CRC cell growth by suppressing apoptosis. Because the association of LUNAR1 was found to be associated with tumour aggressiveness in clinical specimens, 


\begin{tabular}{|l|l|l|l|l|}
\hline & Unadjusted HR* $\mathbf{9 5} \% \mathbf{C I})$ & $\boldsymbol{P}$ & ${\left.\text { Adjusted } \mathbf{H R}^{\dagger} \mathbf{( 9 5} \% \mathbf{C I}\right)}$ & $\boldsymbol{P}$ \\
\hline LUNAR1 level & $3.25(1.98-5.31)$ & $<0.001$ & $2.81(1.69-4.67)$ & $<0.001$ \\
\hline Sex & $0.92(0.697-1.40)$ & 0.983 & $0.91(0.63-1.32)$ & 0.630 \\
\hline Age & $1.24(0.87-1.77)$ & 0.238 & $1.03(0.71-1.49)$ & 0.894 \\
\hline Tumour location & $1.11(0.53-2.32)$ & 0.790 & $1.18(0.79-1.77)$ & 0.406 \\
\hline Tumour size & $1.82(0.56-5.95)$ & 0.319 & $1.50(0.48-4.67)$ & 0.489 \\
\hline Differentiation status & $2.44(1.45-4.11)$ & 0.001 & $1.19(0.36-3.95)$ & 0.780 \\
\hline TNM stage & $3.01(1.60-5.66)$ & 0.001 & $3.26(1.71-6.20)$ & $<0.001$ \\
\hline
\end{tabular}

Table 2. Association of LUNAR1 and clinical factors with disease-free survival in patients with CRC. *Hazard ratios in univariate models. ${ }^{\dagger}$ Hazard ratios in multivariable models. Abbreviations: HR, hazard ratio; 95\% CI, 95\% confidence interval.

\begin{tabular}{|l|l|l|l|l|}
\hline & Unadjusted HR* $\mathbf{9 5} \% \mathbf{C I})$ & $\boldsymbol{P}$ & Adjusted HR $^{\dagger}$ (95\% CI) & $\boldsymbol{P}$ \\
\hline LUNAR1 level & $3.49(1.98-6.14)$ & $<0.001$ & $2.79(1.56-4.99)$ & 0.001 \\
\hline Sex & $0.98(0.67-1.45)$ & 0.942 & $0.89(0.60-1.34)$ & 0.584 \\
\hline Age & $1.12(0.76-1.64)$ & 0.572 & $1.11(0.75-1.65)$ & 0.607 \\
\hline Tumour location & $1.25(0.82-1.73)$ & 0.763 & $1.03(0.65-1.69)$ & 0.902 \\
\hline Tumour size & $1.53(0.98-2.39)$ & 0.061 & $1.26(0.59-2.71)$ & 0.511 \\
\hline Differentiation status & $2.13(1.23-3.68)$ & 0.007 & $1.16(0.63-2.15)$ & 0.626 \\
\hline TNM stage & $3.19(1.43-7.14)$ & $<0.001$ & $3.54(1.54-8.14)$ & 0.003 \\
\hline
\end{tabular}

Table 3. Association of LUNAR1 and clinical factors with overall survival in patients with CRC. *Hazard ratios in univariate models. ${ }^{\dagger}$ Hazard ratios in multivariable models. Abbreviations: HR, hazard ratio; 95\% CI, 95\% confidence interval.

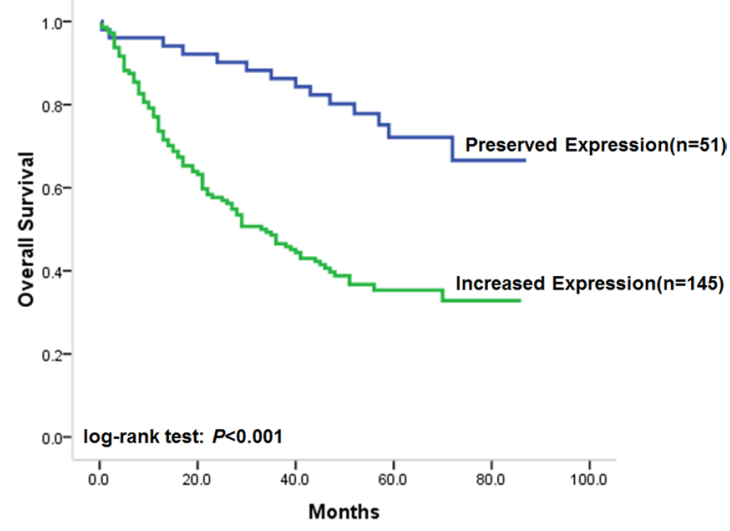

Figure 3. Correlation of LUNAR1 expression with overall survival. Kaplan-Meier univariate survival analysis showed that preserved LUNAR1 expression in CRC was significantly associated with favorable OS of patients.

we next performed a transwell assay to confirm the effect of LUNAR1 on the migration and invasion of cells. The results showed that downregulation of LUNAR1 attenuated the capacity of migration and invasion in SW620 cells (Fig. 4E). To validate the enhancing effect of LUNAR1 on IGF1 signalling, we next evaluated the expression alteration of IGF1R after the downregulation of LUNAR1. The results showed that IGF1R was consequently decreased in LUNAR1-interfered SW620 cells (Fig. 4F). To further convince that LUNAR1 contributes to CRC progression through regulation of IGF1 signal in a rescue manner. We performed RNAi mediated depletion of LUNAR1 in CRC cells followed by ectopically expressing of IGF1R (Fig. 4F). Results on CRCs proliferation showed that ectopically IGF1R expression after LUNAR1 interference reversed the results of LUNAR1 siRNA transfection (Fig. 4G).

\section{Discussion}

$\mathrm{CRC}$ is one of the most common malignant tumours worldwide. Even after surgical resection and adjuvant therapy, the prognosis of CRC is still unfavourable. Therefore, the accurate prediction of prognosis is necessary to better and more precisely manage $\mathrm{CRC}^{13,14}$. In addressing this challenge, our present study provided novel insight to explore new potential molecular markers for prognosis predication and as measures to evaluate the therapeutic effectiveness of medical interventions ${ }^{15,16}$. 
A

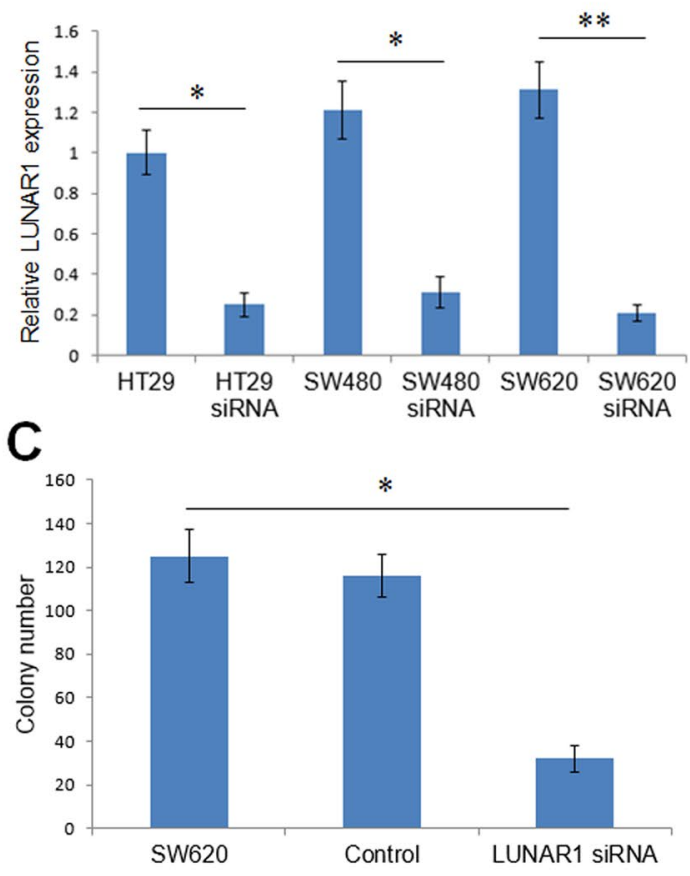

$E$

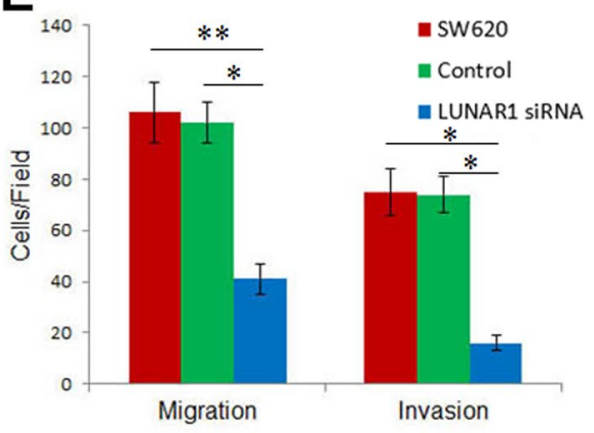

B
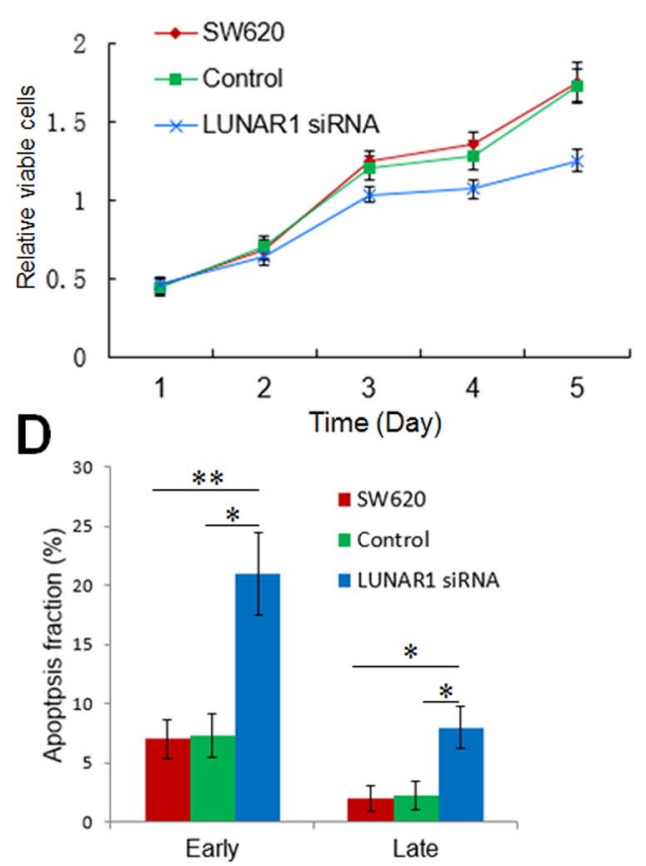

$\mathbf{F}$

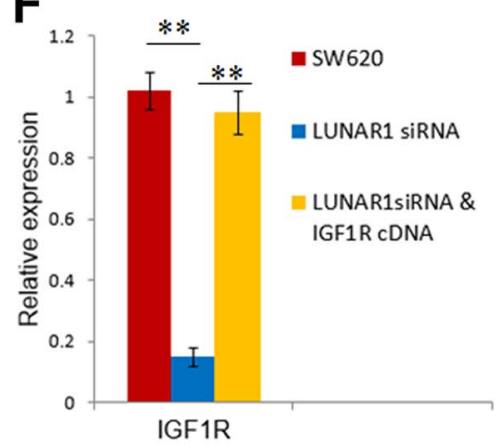

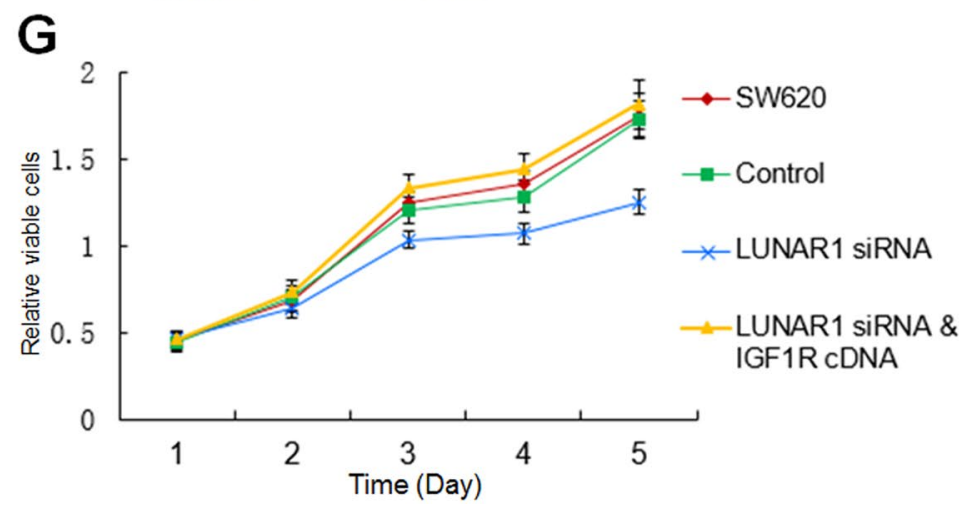

Figure 4. Inhibition of LUNAR1 suppresses the proliferation and invasion of colorectal cancer cells via IGF1 signalling. (A) Downregulation of LUNAR1 by siRNA verified by real-time PCR. The relative expression of LUNAR1 was analyzed using the $2^{-\Delta \Delta C t}$ method. (B) The proliferation of cells detected by CCK- 8 reagent. The data represent the results of three independent experiments. (C) Colony formation assay of three independent experiments. (D) The percentage of early apoptotic cells (Annexin V-positive and PI-negative) and late apoptotic cells (Annexin V-positive and PI-positive); the data represent the results of three independent experiments. (E) Migration and invasion assays; the counts of cells are presented as the mean values per field from at least five randomly-selected low-powered fields $(\times 200)$ from three independent experiments (error bars: Means \pm SDs). (F) IGF1R expression was decreased after LUNAR1 siRNA transfection, and increased after ectopically expressing vector containing IGF1R; (G) The proliferation of CRC cells detected by CCK-8 reagent indicated that ectopically IGF1R expression after LUNAR1 interference reversed the results of LUNAR1 siRNA transfection alone, the data represent the results of three independent experiments. $(* P<0.05, * * P<0.001)$. 
The potential function and clinical utility of the Notch signal-induced long noncoding RNA LUNAR1 in $\mathrm{CRC}$ is the primary purpose of the present investigation. Considering the paradox of the complex function and simplicity of the target gene, we assume that this Notch signal-induced lncRNA might play significant roles in CRC. The investigation of LUNAR1 expression in clinical specimens indicated that its level in CRC was significantly increased compared with that in adjacent normal specimens. Moreover, the increased expression pattern of LUNAR1 was more likely to be detected in tumours with a poor differentiation status or an advanced TNM stage. These results suggested that LUNAR1 might act as an oncogene and play a significant role in cancer progression. Therefore, elucidating whether LUNAR1 expression in CRC could predict the DFS and OS of patients with CRC could further determine its potential clinical utility. Next, we conducted univariate and multivariate survival analyses to evaluate the prognostic role of LUNAR1 in CRC. The Kaplan-Meier analysis and Cox proportional hazards model analysis resultssuggested that increased LUNAR1 expression levels in CRC were independently associated with unfavourable DFS and OS outcomes after controlling for known prognostic factors. In this context, LUNAR1 might constitute an independent biomarker of tumour recurrence and prognosis for CRC patients and could contribute to precise diagnosis and treatment. As LUNAR1 is a Notch signal-induced lncRNA, our results also indicated that the function of LUNAR1 could be a part of Notch signalling. To explore the cellular mechanism of this Notch-induced lncRNA, we conducted an investigation using LUNAR1 interference in colorectal cell lines. The results showed that the proliferation, colony formation and invasion of colorectal cancer cells were significantly inhibited after the downregulation of LUNAR1, while cell apoptosis was induced. We also performed RNAi mediated depletion of LUNAR1 in CRC cells followed by ectopically expressing of IGF1R, in order to confirm the mechanism of LUNAR1 in a rescue manner. Results of CRC cells proliferation showed that ectopically IGF1R expression after LUNAR1 interference reversed the results of LUNAR1 siRNA transfection, indicating that LUNAR1 contributes to the CRC progression through regulation of IGF1 signal. These results further support the oncogenic role of LUNAR1 in colorectal cancer.

Recently, a series of investigations demonstrated that lncRNAs are aberrantly expressed in various human malignancies ${ }^{17,18}$. Given the complex roles of Notch signalling and its few known direct target genes, this lncRNA might be a potential functional target of the Notch pathway. We sought to determine whether LUNAR1, a Notch-induced lncRNA first reported in T-ALL, plays a significant role in colorectal cancer. Our investigation revealed that LUNAR1 siRNA transfection could inhibit IGF1R expression, confirming the sustaining effect of LUNAR1 on IGF1 signalling. Accumulating evidence has suggested that the over-activation of IGF1 signalling contributes to the development and progression of colorectal cancer ${ }^{19}$.

The present study, including clinical specimens and cell lines, provided the first piece of evidence suggesting that the increased LUNAR1 expression pattern in CRC may serve as an independent predictor of tumour recurrence and prognosis through its regulation of proliferation, indicating that the function of the lncRNA-mediated non-canonical pathway constitutes part of the Notch signal in CRC.

\section{Methods}

Clinical specimens. The protocols utilized in this study were approved by the ethics committees of Xi'an Jiaotong University and the Fourth Military Medical University. All patients recruited in this study provided informed consent. All research was performed in accordance with the relevant guidelines and regulations. The participants were randomly recruited from those who were consecutively diagnosed with CRC and underwent surgical resection between February 2010 and November 2012 at the Xijing Hospital of Digestive Diseases, the Fourth Military Medical University (Xian, China). Clinical CRC specimens were collected by surgeon in $10 \mathrm{~min}$ after surgical resection and put into liquid nitrogen for $10 \mathrm{~min}$, then into a $-80^{\circ} \mathrm{C}$ ultra-freezer for mRNA isolation. The histology of these specimens had been double checked and confirmed by pathologists. The clinicopathological data and follow-up information of the participants were prospectively entered into a database, which was updated according to the survival status of participants by trained nurses every six months via telephone interview or questionnaire letters. Patients who had invalid census data, non-adenocarcinoma, inaccurate follow-up, missing data regarding pathological information or withdrew from the survey were excluded.

Study endpoints. Disease-free survival (DFS) was defined as the time elapsed from surgery to the first occurrence of any of the following events: CRC distant metastasis, recurrence of CRC, or the development of a second non-colorectal malignancy excluding basal cell carcinomas of the skin and carcinoma in situ of the cervix; or death from any cause without documentation of a cancer-related event. The diagnosis of recurrence and distant metastasis was based on imaging methods such as ultrasonography, computed tomography, magnetic resonance imaging and position emission tomography and, if possible, cytologic analysis or biopsy. Overall survival (OS) was defined as the time elapsed from surgery to death of patients with CRC.

Real-time PCR, mutation and MSI investigation. Total RNA from clinical CRC specimens and normal tissue specimens was purified using Trizol reagent (Invitrogen, Carlsbad, CA) according to the manufacturer's instructions. cDNA synthesis was performed with $5 \mu \mathrm{g}$ of total RNA per $20 \mu \mathrm{L}$ using a cDNA reverse transcription kit (Fermentas, Lithuania). Real-time PCR was carried out on an ABI 7700 system (Applied Biosystems) using SYBR Green I (Invitrogen, Carlsbad, CA) as described before ${ }^{20}$. The primers for LUNAR1 were forward 5'-GGAGGCTGAGGCCGCCTGTT-3' and reverse 5'-AGGCTGCAGGGGAACAGGTCTT-3'. The IGF1R primers were forward $5^{\prime}$-TACTCGGACGTCTGGTCCTT- $3^{\prime}$ and reverse $5^{\prime}$-TGGGGTTATACTGCCAGCAC- $3^{\prime}$. The internal control $18 \mathrm{~S}$ rRNA primers were forward $5^{\prime}$-CGCCGCTAGAGGTGAAATTC-3' and reverse $5^{\prime}$-TTGGCAAATGCTTTCGCTC-3'. The relative expression of target gene, normalized to $18 \mathrm{~S}$ rRNA, was analyzed using the $2^{-\Delta \Delta C t}$ method. DNA extraction, microsatellite instability (MSI) analysis, and the pyrosequencing of KRAS, BRAF and PIK3CA were performed as described before ${ }^{21-23}$. 
Cell Lines and transfection. Colorectal cancer cell lines utilized in the present study are HT29, SW480 and SW620, which was purchased from the Chinese Academy of Sciences Cell Bank (Shanghai, China). According to the instructions for these cell lines, the cells were cultured in L-15 or 1640 medium (Invitrogen, Carlsbad, CA) supplemented with $10 \%$ foetal calf serum. To downregulate LUNAR1 expression in cells, LUNAR1 targeted stealth RNAi (LUNAR1 siRNA) was utilized (Invitrogen, Carlsbad, USA). The sequence of the LUNAR1-targeted small interfering RNA was GATTCTGTAGCCTAACAAGCTTTCCAGTGATT. During transfection, according to the manufacturer's instructions, the cells were transfected with $50 \mathrm{nmol}$ of LUNAR1 siRNA using Lipofectamine 2000 transfection reagent (Invitrogen, Carlsbad, USA) with a scrambled negative control. After transfection of LUNAR1 siRNA, cells were also transfected with plasmids containing IGF-1R (pcDNA3.1-IGF1R) and empty vector (GenePharma, Shanghai, China). The transfected cells were harvested after $48 \mathrm{~h}$ of incubation for real-time PCR investigation to evaluate the transfection efficiency and target gene expression.

Cell viability, colony formation and apoptosis assays. The Cell Counting Kit-8 (Dojindo, Kumamoto, Japan) was utilized to evaluate cell proliferation by counting viable cells. After screening, LUNAR1 siRNA stably transfected cells and controls were plated into 96-well plates. During an incubation of 5 consecutive days, the culture medium for both stably transfected cells and controls was changed and replenished with $10 \mu \mathrm{l}$ of CCK- 8 reagent and $90 \mu \mathrm{l}$ of fresh L-15 medium. Then, after incubation at $37^{\circ} \mathrm{C}$ for $1 \mathrm{~h}$, the 96 -well plates were evaluated by a microplate reader (Pharmacia Biotech) according to the manufacturer's instructions. In addition, in the colony formation assay, LUNAR1 siRNA stably transfected cells and controls were plated into 6-cm plates (200 cells per plate) and cultured in L-15 medium (Invitrogen, Carlsbad, CA) with 10\% foetal calf serum. After 2 weeks, the colonies in the plates were fixed with cold methanol and stained with $1 \%$ crystal violet. The FITC Annexin V Apoptosis Detection Kit (BD Pharmingen, USA) was utilized to evaluate the difference in apoptosis between LUNAR1 siRNA-transfected cells and controls by flow cytometry. LUNAR1 siRNA-transfected cells and controls were harvested every $12 \mathrm{~h}$ within $48 \mathrm{~h}$ after transient transfection and washed in PBS and then stained with Annexin V and propidium iodide. The percentage of apoptotic cells in the LUNAR1 siRNA-transfected cells and controls was quantified by a BD FACS Verse flow cytometer. Each experiment above was repeated in triplicate.

Migration and invasion assays. Transwell experiments were utilized for the evaluation of migration and invasion. Specifically, 8- $\mu \mathrm{m}$ transwell polycarbonate insert chambers (BD Biosciences, San Jose, CA) coated with and without $40 \mu \mathrm{l}$ of Matrigel (Corning, NY, CA) were prepared. Then, the chambers were incubated at $37^{\circ} \mathrm{C}$ for $2 \mathrm{~h}$ to solidify the Matrigel. Afterwards, the same density $\left(1 \times 10^{5} \mathrm{in} 100 \mu \mathrm{l}\right)$ of LUNAR1 siRNA-transfected cells and control cells in either serum-free DMEM/F12 or DMEM-high glucose were added into the upper compartment of the transwell polycarbonate insert chambers. In the bottom chambers, $600 \mu \mathrm{l}$ of conditioned medium for cell use was placed as a chemoattractant. The medium in the upper chamber was removed after incubation at $37^{\circ} \mathrm{C}$ and $5 \% \mathrm{CO}_{2}$ for $24 \mathrm{~h}$. The invaded LUNAR 1 siRNA-transfected cells and control cells in the lower membrane were fixed with $4 \%$ paraformaldehyde and stained with $0.1 \%$ crystal violet (Invitrogen, Carlsbad, CA).

Statistical analysis. SPSS (version 16.0) software was utilized for statistical analysis. The associations of LUNAR1 levels with clinicopathological characteristics were investigated by the Pearson $\chi^{2}$ test or Fisher's exact test. Kaplan-Meier univariate survival analysis was utilized to identify potential prognostic factors for the survival of CRC patients. In the multivariate Cox proportional hazards model analysis, independent prognostic factors were justified by adjusting for known prognostic factors. Statistically significant differences were defined as a $P$ value of 0.05 or less.

Received: 11 April 2019; Accepted: 10 December 2019;

Published online: 27 December 2019

\section{References}

1. Zlobin, A., Jang, M. \& Miele, L. Toward the rational design of cell fate modifiers: notch signaling as a target for novel biopharmaceuticals. Current pharmaceutical biotechnology 1, 83-106 (2000).

2. Mumm, J. S. \& Kopan, R. Notch signaling: from the outside in. Developmental biology 228, 151-165, https://doi.org/10.1006/ dbio.2000.9960 (2000).

3. Weng, A. P. \& Aster, J. C. Multiple niches for Notch in cancer: context is everything. Current opinion in genetics \& development 14, 48-54, https://doi.org/10.1016/j.gde.2003.11.004 (2004).

4. Bray, S. J. Notch signalling: a simple pathway becomes complex. Nature reviews. Molecular cell biology 7, 678-689, https://doi. org/10.1038/nrm2009 (2006).

5. Leong, K. G. \& Karsan, A. Recent insights into the role of Notch signaling in tumorigenesis. Blood 107, 2223-2233, https://doi. org/10.1182/blood-2005-08-3329 (2006).

6. Fre, S. et al. Notch and Wnt signals cooperatively control cell proliferation and tumorigenesis in the intestine. Proceedings of the National Academy of Sciences of the United States of America 106, 6309-6314, https://doi.org/10.1073/pnas.0900427106 (2009).

7. van Es, J. H. et al. Notch/gamma-secretase inhibition turns proliferative cells in intestinal crypts and adenomas into goblet cells. Nature 435, 959-963, https://doi.org/10.1038/nature03659 (2005).

8. Chu, D. et al. Notch1 expression in colorectal carcinoma determines tumor differentiation status. Journal of gastrointestinal surgery: official journal of the Society for Surgery of the Alimentary Tract 13, 253-260, https://doi.org/10.1007/s11605-008-0689-2 (2009).

9. Chu, D. et al. High level of Notch1 protein is associated with poor overall survival in colorectal cancer. Annals of surgical oncology 17, 1337-1342, https://doi.org/10.1245/s10434-009-0893-7 (2010).

10. Chu, D. et al. Notch2 expression is decreased in colorectal cancer and related to tumor differentiation status. Annals of surgical oncology 16, 3259-3266, https://doi.org/10.1245/s10434-009-0655-6 (2009).

11. Chu, D. et al. Notch1 and Notch2 have opposite prognostic effects on patients with colorectal cancer. Annals of oncology: official journal of the European Society for Medical Oncology 22, 2440-2447, https://doi.org/10.1093/annonc/mdq776 (2011).

12. Trimarchi, T. et al. Genome-wide mapping and characterization of Notch-regulated long noncoding RNAs in acute leukemia. Cell 158, 593-606, https://doi.org/10.1016/j.cell.2014.05.049 (2014). 
13. Halabi, S. et al. Updated Prognostic Model for Predicting Overall Survival in First-Line Chemotherapy for Patients With Metastatic Castration-Resistant Prostate Cancer. Journal of clinical oncology: official journal of the American Society of Clinical Oncology, https:// doi.org/10.1200/JCO.2013.52.3696 (2014).

14. Hehlmann, R. et al. Deep Molecular Response Is Reached by the Majority of Patients Treated With Imatinib, Predicts Survival, and Is Achieved More Quickly by Optimized High-Dose Imatinib: Results From the Randomized CML-Study IV. Journal of clinical oncology: official journal of the American Society of Clinical Oncology 32, 415-423, https://doi.org/10.1200/JCO.2013.49.9020 (2014).

15. Ferrari, A. et al. Comparison of the prognostic value of assessing tumor diameter versus tumor volume at diagnosis or in response to initial chemotherapy in rhabdomyosarcoma. Journal of clinical oncology: official journal of the American Society of Clinical Oncology 28, 1322-1328, https://doi.org/10.1200/JCO.2009.25.0803 (2010).

16. Bulian, P. et al. CD49d Is the Strongest Flow Cytometry-Based Predictor of Overall Survival in Chronic Lymphocytic Leukemia. Journal of clinical oncology: official journal of the American Society of Clinical Oncology, https://doi.org/10.1200/JCO.2013.50.8515 (2014).

17. Du, Z. et al. Integrative genomic analyses reveal clinically relevant long noncoding RNAs in human cancer. Nature structural \& molecular biology 20, 908-913, https://doi.org/10.1038/nsmb.2591 (2013).

18. Prensner, J. R. et al. The long noncoding RNA SChLAP1 promotes aggressive prostate cancer and antagonizes the SWI/SNF complex. Nature genetics 45, 1392-1398, https://doi.org/10.1038/ng.2771 (2013).

19. Pollak, M. Insulin and insulin-like growth factor signalling in neoplasia. Nature reviews. Cancer 8, 915-928, https://doi.org/10.1038/ nrc2536 (2008).

20. Sanders, R., Mason, D. J., Foy, C. A. \& Huggett, J. F. Considerations for accurate gene expression measurement by reverse transcription quantitative PCR when analysing clinical samples. Analytical and bioanalytical chemistry 406, 6471-6483, https://doi. org/10.1007/s00216-014-7857-x (2014).

21. Ogino, S. et al. Sensitive sequencing method for KRAS mutation detection by Pyrosequencing. The Journal of molecular diagnostics: JMD 7, 413-421, https://doi.org/10.1016/S1525-1578(10)60571-5 (2005).

22. Ogino, S., Kawasaki, T., Kirkner, G. J., Loda, M. \& Fuchs, C. S. CpG island methylator phenotype-low (CIMP-low) in colorectal cancer: possible associations with male sex and KRAS mutations. The Journal of molecular diagnostics: JMD 8, 582-588, https://doi. org/10.2353/imoldx.2006.060082 (2006)

23. Nosho, K. et al. PIK3CA mutation in colorectal cancer: relationship with genetic and epigenetic alterations. Neoplasia 10, 534-541 (2008).

\section{Acknowledgements}

The present study is supported by grants from the National Natural Science Foundation of China (Nos 81201927 and 81672460), Innovation Talent Promotion Plan of Shaanxi (No. 2017KJXX-07) and Key Research and Development Program of Shaanxi Province (No. 2019SF-012). The funders had no role in the design of the study, the collection, analysis, or interpretation of the data, the writing of the manuscript, or the decision to submit the manuscript for publication. There are no potential conflicts of interest to disclose.

\section{Author contributions}

D.K.C. and W.Z.W. conceived and designed the study. Z.X.Z., X.B., S.J.Z. and C.X.D. were responsible for the clinical data extraction. Z.X.Z., G.L., H.Q., J.Y.Y., X.B. and J.Y.Z. were responsible for experimental preformation, collection and/or assembly of data, data analysis and interpretation. Z.X.Z. and D.K.C. wrote the manuscript. C.X.D. and W.Z.W. involved in manuscript revision. All authors read and approved the final manuscript.

\section{Competing interests}

The authors declare no competing interests.

\section{Additional information \\ Correspondence and requests for materials should be addressed to D.C.}

Reprints and permissions information is available at www.nature.com/reprints.

Publisher's note Springer Nature remains neutral with regard to jurisdictional claims in published maps and institutional affiliations.

Open Access This article is licensed under a Creative Commons Attribution 4.0 International License, which permits use, sharing, adaptation, distribution and reproduction in any medium or format, as long as you give appropriate credit to the original author(s) and the source, provide a link to the Creative Commons license, and indicate if changes were made. The images or other third party material in this article are included in the article's Creative Commons license, unless indicated otherwise in a credit line to the material. If material is not included in the article's Creative Commons license and your intended use is not permitted by statutory regulation or exceeds the permitted use, you will need to obtain permission directly from the copyright holder. To view a copy of this license, visit http://creativecommons.org/licenses/by/4.0/.

(C) The Author(s) 2019 